\title{
Evidence Linking Stunting to Economic Outcomes in the SAARC Region: a Systematic Review
}

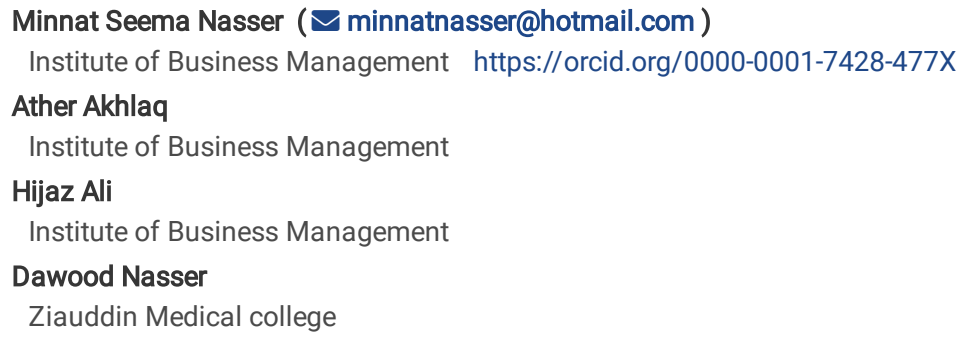

Systematic Review

Keywords: Economic Outcomes, Poverty, Review, SAARC Countries, Stunting

Posted Date: February 8th, 2022

DOl: https://doi.org/10.21203/rs.3.rs-1330705/v1

License: 두 (i) This work is licensed under a Creative Commons Attribution 4.0 International License. Read Full License 


\section{Abstract \\ Background}

At present, South Asian Association for Regional Coorporation (SAARC) countries have tremendous pressure on child stunting along with the impact it is making on economic growth. World Health Organization (WHO), United Nations International Children's Emergency Fund (UNICEF) and The World Bank (WB) have emphasized for this region to reduce stunting by $40 \%$. The objective of this paper is to analyzed the impact of stunting on economic outcomes in SAARC countries so that to scale up policies and programs aiming to reduce child stunting.

\section{Methods}

The systematic review has assessed five international databases; PubMed, JSTOR, Cochrane Library, Web of Science and CINAHL Plus for published, unpublished, and on-going research till the year 2020. Grey literature is searched using Google Scholar and Google search engines. The systematic review registration number in PROSPERO is CRD42021230279.

\section{Result}

Thirty-three studies matched the criteria. Most quantitative studies link stunting with economic repercussions. Three investigations found no or a weak link between the two. Nutritional intervention synthesis predicts a 12 percent return, a 5:1-6:1 benefit-cost ratio, and a 14mm height gain. A $1 \mathrm{~cm}$ increase in height increases pay by $4 \%$ for men and $6 \%$ for women. Stunting caused a $5 \%-7 \%$ income penalty and lower lifetime pay. Studies show that a $10 \%$ increase in agricultural growth reduces stunting by $9.6 \%$, while a $10 \%$ increase in non-agricultural growth reduces stunting by $8.4 \%$.

\section{Conclusion}

To reduce stunting prevalence, policies should be scaled up, as well as targeted and structural interventions are needed.

\section{1- Introduction}

According to the WHO (1), globally in 2020, 149 million children under the age of five suffered from stunting, which causes them to be too short for their age and more than two standard deviations below the WHO's median (a value of a child's height in the middle of a frequency distribution of observed values of average child height, with an equal probability of falling above or below) (2). South Asia alone accounts for 53.8 million (31.8\%) of the total (1). Stunting is caused by poverty, malnutrition, and chronic disease in the mother or child by birth. Stunting has immediate and intermediate causes, as well as underlying factors (3).

Inadequate nutrition and repeated illnesses in the first five years of a child's life result in decreased IQ and physical development, reduced productivity, and poor health in general, which eventually cause chronic diseases including arthritis, asthma, cancer, and diabetes (3). Stunted children are less productive; mentally, physically, and physiologically (4), which results in lower wages and a likely contender for the next generation/s to be stunted. Stunting also reduces immunity, making people more susceptible to illnesses and affecting reproductive health $(5,6)$. Due to health concerns, stunted children suffer significant mental and physical effects in their early years, resulting in poor performance in school or in vocational training, poor job performance, lower wages and earnings due to lack of education and skills, which causes them to rely on other household members or society to sustain economic burden (5). Stunting has a lifelong impact (7). Height in childhood determines adulthood height (7), and height is an important determinant of wages (5, 8). As reported by (9-11) a child's first 1000 days including enneadic months in the mother's womb impacts the future height. It is difficult to catch up on height later in adolescence due to a malnourished skeleton that takes time to grow taller than the usual non-stunted child $(12,13)$.

Malnutrition is rampant in South Asia, as adequate food is out of reach for many poor people. They also lack the knowledge or education to make healthy food choices (14). According to World Bank's report, 62 million children out of 155 million are considered stunted, meaning they are too small for their age (15). According to the WHO, what sets South Asia apart from other developing countries where stunting is a problem is the persistence of chronic malnutrition notwithstanding rapid economic growth (16-22). This is due to poor transition mechanisms and weak supply side by countries in terms of inadequate and non-nutritious food, dirty water, poor sanitation, insufficient healthcare, to name a few examples. Due to poverty and its related reasons, the demand side is also weak; underinvesting in healthy food, education, old and traditional belief systems (like gender-based discrimination and the waste of colostrum), are a few of them.

\section{2- Method}

This study assessed the economic consequences of stunting in SAARC, considering the impact of childhood stunting on adult economic outcomes. Terms like malnutrition, under-nutrition, and other such categories are used in this study as substitutes for stunting. The PICO questions were used to construct the protocol for this systematic review.

\subsection{Eligibility Criteria (PICO) Eligible population}


Stunted children five or under five years of age

\section{Eligible interventions}

Malnutrition in mother and child, height is to wage, GDP, income

Eligible comparator

Stunted vs non-stunted children.

\section{Outcomes}

Primary Outcomes

impact of stunting on income and economic growth.

\section{Secondary outcome}

causal relationship between economic growth and stunting.

\section{(Detail of the PICO criteria is available in Appendix I)}

This systematic review searched all published, unpublished, and working papers on stunting and its economic consequences up to May 2020 . Studies on Afghanistan, Bangladesh, Bhutan, Maldives, Nepal, India, Pakistan, and Sri Lanka are chosen. We used CINAHL Plus, Cochrane Library, JSTOR, PubMed, and Web of Science to find articles. We used Google Scholar and Chrome to find grey literature and used the top 100 results. The PRISMA guidelines are followed.

\subsection{Inclusion/Exclusion criteria}

The study included all journals, articles, or books on stunting or terms associated with stunting in relation to the economy, adulthood income, and GDP. We did not include studies directly on stunting that have an economic impact due to education, cognition, test scores, health, socioeconomic position, micronutrient deficiencies, and fetal development. We also included research on the causal relationship between GDP and socioeconomic growth via interventions and policy instruments in health education and socioeconomic relevance.

\subsection{Search strategy}

The terms were searched using Boolean methodology. Appendix A. details the search approach for databases.

\subsection{Study selection}

MSN and HA separately incorporated the retrieved studies. The studies were de-duplicated and stored in EndNote. The writers included papers that reported evidence of economic growth and stunting. Studies that did not cover the SAARC region or that sought other outcomes than economic growth were also omitted. The articles were moved to an EndNote library to be searched for duplicates. Author, year of publication, total number of observations, location of study, study design, duration of study, gender, nutritional status, types of nutrition/nutrients supplement, exposure group, result and focus view, economic outcomes (tables vary with headings according to the study design requirement). Fig. 1 shows the article selection procedure in detail.

\subsection{Risk of bias (quality) assessment}

The authors independently screened all titles, abstracts, and full-text papers included in the review. Any arguments were discussed with third team member Ather Akhlaq (AA) and resolved amicably. The authors utilized (23)'s Mixed Method Assessment Tool (MMAT) to measure quality. Many systematic reviews employ this tool $(24,25)$. The whole text of the recovered and synthesized papers is tabulated as in Appendix B, C, D, E, F, G. Each study is given a methodological rating of $0,25,50,75$, or 100 (100 being the greatest quality). With the MMAT, we rated each method separately. When process evaluation was built into the study design, the overall study quality was assessed.

\subsection{Study registration:}

A registration of the study is available at: http://www.crd.york.ac.uk/PROSPERO/display record.asp?ID= CRD42021230279.

\section{3- Result}

The five databases plus Google Scholar retrieved 9689 articles. 6523 articles remained after 3166 publications were de-duplicated. 6050 items were filtered. 258 publications were omitted from the remaining 473 abstracts. Professors; Borghi, Aguayo and Zulfiqar Ali Bhutta were all provided with the reference list for their expert advice. Dr. Elaine Borghi contributed nine publications, including editorials and research, six were included in our references. Dr. Zivai Murira responded on Dr. Aguayo's behalf and offered one article. Dr. Bhutta did not respond to our second and third soft reminder emails, 10 days apart. We evaluated the remaining 221 publications closely after adding Dr. Borgi's six articles. 29 items qualified. 4 articles were added after reading references from retrieved articles. Figure 1 displays the results of the search of the selected publications' bibliographies.

The link between child stunting and economic outcomes was studied despite the lack of data. We took 19 global studies where one or more SAARC countries were a part of them. Our review includes news, journal, and report articles. Our research found seven papers on India and two news reports on Pakistan. One interview, one cover story, and one study on stunting in D/LMIC countries are included in this review. We found 15 quantitative, 11 qualitative, and 7 mixedmethod studies. 
Quality appraisa/ Overall, the studies that were included were of high quality. 25 of the 33 full research papers evaluated received a quality score of equal to or greater than $75 \%$. Quantitative studies had the best quality score of all sorts of methodologies, followed by qualitative studies and mixed methods research (see Table 1).

Table 1

\begin{tabular}{|lllll|}
\hline Study design & \multicolumn{4}{c|}{ Number of studies and methods used to collect data } \\
\hline Study design & $25 \%(*)$ & $50 \%(* *)$ & $75 \%(\star \star *)$ & $100 \%(\star \star \star \star)$ \\
\hline Quantitative & & 2 & 7 & 6 \\
\hline Qualitative & 1 & 2 & 4 & 4 \\
\hline Mixed Methods & 1 & 2 & 3 & 1 \\
\hline
\end{tabular}

Synthesis of results The themes of study types indicating the link between child stunting and economic outcomes in the SAARC region were intervention studies, natural experiments, prospective studies, quasi-experiments, and linear regression estimations. Evidence identified under these study types is presented in Tables 3, 4, 5, 6, 7. In addition to the study types, the link between stunting and economic outcomes is also mentioned, with supporting key background papers as presented in Table 2 .

\subsection{Reasons for stunting and its Economic outcomes:}

Table 2. includes the twenty background articles that outline the reasons for stunting and its economic consequences in the SAARC Region. Stunting in the first two years of life or the first 1000 days leads to poorer human capital $(11,26)$. It also causes persistent damage that hinders an individual's economic growth $(11,26)$. This harm impacts the next generation, both physically and financially $(11,26)$. Paternal stimulation was also found to be a factor in a child's physical and mental development (27). Rudra and Kurian (2017) evaluated the impact of stunting and malnutrition on India's workforce and found that stunted children earn $20 \%$ less than non-stunted children in adulthood, which impacts the national income and causes irreversible damage (28). A study in Bangladesh, India, Nepal, Pakistan, Sri Lanka, Bhutan, and 85 other LMICs indicated that stunting has a long-term influence on human development (29). Stunting reduces output and is validated by UNFP (2017). The paper states that stunting, anemia, and iodine deficiency affect more than two-thirds of Pakistani children, resulting in mental and physical development deficits, lower school performance, fatigue and increased mortality. The cost to the economy due to this reason is around UD $\$ 657$ million annually in industry, agriculture, and other physical jobs (30).

Stunting and poverty are inextricably linked (31). Evidence-based research shows that poverty puts 65 percent of South Asian children at risk of stunting. According to Harvard research, stunting can cost developing countries like South Asia over $\$ 177$ billion in lifetime earnings. According to an Indian study on stunting and height (5) a $1 \mathrm{~cm}$ increase in height results in a $4 \%$ increase in male wage and a $6 \%$ increase in female wage. Stunting can be reduced by improving children's nutrition, addressing gender gaps and enhancing women's status, improving sanitation, and lowering poverty and unfairness. Stunting or short stature in childhood is frequently linked to low adult economic performance (5). According to (32), stunting and micronutrient deficiencies cost lowincome nations, particularly South Asia, 3\% of their GDP. Economic shocks have a pro-cyclical influence on health in Asia (33). Part of the current workforce was stunted in childhood $(8,34)$, resulting in a loss to national economic productivity (35) In the cases of India, Pakistan, and Bangladesh, despite being among the world's most populous countries, economic growth has been uneven in the last 15-20 years due to low labor productivity (12). A study (19) discovered that economic growth had little impact on stunting because of poor government strategies, an unfavorable environment (36), ineffective interventions (37), and a lack of integrated and structural policies (22). 
Table 2

Reasons for stunting and its Economic outcomes

\begin{tabular}{|c|c|c|c|c|c|c|}
\hline Author's Name & Publication & $\begin{array}{l}\text { Year of } \\
\text { publication }\end{array}$ & $\begin{array}{l}\text { Studied Country/ies } \\
\text { Prevailing } \\
\text { Circumstances }\end{array}$ & Outcomes & $\begin{array}{l}\text { Effect on } \\
\text { Economic } \\
\text { growth }\end{array}$ & Focus View \\
\hline $\begin{array}{l}\text { Cesar G } \\
\text { Victora et al. }\end{array}$ & Lancet & 2008 & $\begin{array}{l}\text { Brazil, Guatemala, India, } \\
\text { the Philippines, and } \\
\text { South Africa. } \\
\text { Undernutrition of } \\
\text { mother and child }\end{array}$ & $\begin{array}{l}\text { School delays, } \\
\text { lower income } \\
\text { and scarce } \\
\text { assets, low } \\
\text { birth weight, } \\
\text { lower body } \\
\text { mass, higher } \\
\text { glucose } \\
\text { concentration } \\
\text { and blood } \\
\text { pressure }\end{array}$ & Adverse & $\begin{array}{l}\text { The damage happened in early life } \\
\text { due to stunting leads to permanent } \\
\text { damage thus affecting economic } \\
\text { growth, and might also affect future } \\
\text { generations. }\end{array}$ \\
\hline Lim et al. & Lancet & 2018 & $\begin{array}{l}195 \text { countries, including } \\
\text { SAARC Countries; India, } \\
\text { Pakistan, and } \\
\text { Bangladesh. } \\
\text { Human capital } \\
\text { investment }\end{array}$ & $\begin{array}{l}\text { Higher } \\
\text { education, } \\
\text { training and } \\
\text { better health }\end{array}$ & $\begin{array}{l}\text { Expansion in } \\
\text { economic } \\
\text { growth }\end{array}$ & $\begin{array}{l}\text { Three countries; India, Pakistan, and } \\
\text { Bangladesh came in } 10 \text { top countries } \\
\text { out of } 195 \text { countries having the } \\
\text { highest human capital but with } \\
\text { marked variation in GDP from a } \\
\text { developed country. }\end{array}$ \\
\hline $\begin{array}{l}\text { Dewey K.G. \& } \\
\text { Begum K. }\end{array}$ & $\begin{array}{l}\text { Maternal and } \\
\text { child } \\
\text { nutrition }\end{array}$ & 2011 & $\begin{array}{l}\text { Brazil, India, Philippines } \\
\text { and South Africa (in } \\
\text { addition to the } \\
\text { Guatemala trial). } \\
\text { Stunting }\end{array}$ & $\begin{array}{l}\text { Impaired } \\
\text { health and } \\
\text { education }\end{array}$ & $\begin{array}{l}\text { Impaired } \\
\text { economic } \\
\text { growth }\end{array}$ & $\begin{array}{l}\text { Summarizes the review on the long } \\
\text { term impact of stunting in later life. }\end{array}$ \\
\hline \multirow[t]{2}{*}{$\begin{array}{l}\text { Emanuela } \\
\text { Galasso \& } \\
\text { Adam } \\
\text { Wagstaff }\end{array}$} & \multirow[t]{2}{*}{$\begin{array}{l}\text { Economics } \\
\text { and Human } \\
\text { Biology; } \\
\text { Elsevier }\end{array}$} & \multirow[t]{2}{*}{2019} & $\begin{array}{l}\text { 1-Stunting } \\
\text { 2-Nutritional } \\
\text { intervention }\end{array}$ & $\begin{array}{l}\text { 1-lesser years } \\
\text { of school and } \\
\text { lower cognitive } \\
\text { skills }\end{array}$ & $\begin{array}{l}\text { 1-lower wages } \\
\text { \& lower } \\
\text { country's per } \\
\text { capita }\end{array}$ & \multirow[t]{2}{*}{$\begin{array}{l}\text { Lower capita in present due to the } \\
\text { fraction of the workforce stunted in } \\
\text { childhood in developing countries that } \\
\text { includes SAARC countries too. }\end{array}$} \\
\hline & & & & $\begin{array}{l}\text { 2-more } \\
\text { schooling and } \\
\text { better } \\
\text { cognition }\end{array}$ & $\begin{array}{l}\text { 2-multiplied rate } \\
\text { of return }\end{array}$ & \\
\hline Emma Batha & Reuters & 2016 & $\begin{array}{l}34 \text { developing countries } \\
\text { including SAARC } \\
\text { countries; Afghanistan, } \\
\text { Bangladesh, India, } \\
\text { Nepal, Pakistan Poverty, } \\
\text { less investment in early } \\
\text { child development, } \\
\text { nutrition, sanitation and } \\
\text { inadequate simulation }\end{array}$ & $\begin{array}{l}\text { Higher health } \\
\text { investment in } \\
\text { later life, } \\
\text { frequent } \\
\text { infections and } \\
\text { low cognition }\end{array}$ & $\begin{array}{l}\text { More poverty, } \\
\text { lower earnings } \\
\text { and low future } \\
\text { economic } \\
\text { growth }\end{array}$ & $\begin{array}{l}\text { Evidence-based study shows that } \\
\text { there is strong evidence linking child } \\
\text { stunting and poverty. } 65 \% \text { of the } \\
\text { children in South Asian states are at } \\
\text { risk of stunting due to poverty. } \\
\text { Harvard study says that developing } \\
\text { countries including south Asia can } \\
\text { lose more than } \$ 177 \text { billion in the } \\
\text { lifetime earning of its people due to } \\
\text { stunting. }\end{array}$ \\
\hline $\begin{array}{l}\text { F. H. G. } \\
\text { Ferreira \& N. } \\
\text { Schady }\end{array}$ & World Bank & 2008 & $\begin{array}{l}\text { India along with other } \\
\text { developing countries } \\
\text { and developed } \\
\text { countries. } \\
\text { Microeconomic crises, } \\
\text { drought and reduced } \\
\text { investment in child } \\
\text { human capital }\end{array}$ & $\begin{array}{l}\text { Lesser school } \\
\text { years, lower } \\
\text { health and } \\
\text { tension } \\
\text { between } \\
\text { income and } \\
\text { substitution } \\
\text { effect }\end{array}$ & $\begin{array}{l}\text { Transmitted } \\
\text { poverty to next } \\
\text { generation, pro- } \\
\text { cyclical } \\
\text { mortality rate, } \\
\text { decrease in } \\
\text { school } \\
\text { enrollment and } \\
\text { fall in nutrition } \\
\text { level }\end{array}$ & $\begin{array}{l}\text { Economic shocks have a pro-cyclical } \\
\text { effect on health in Asia }\end{array}$ \\
\hline Fink G. et al & $\begin{array}{l}\text { American } \\
\text { Journal of } \\
\text { Clinical } \\
\text { Nutrition }\end{array}$ & 2016 & $\begin{array}{l}\text { Nepal, Bhutan, } \\
\text { Bangladesh included in } \\
38 \text { LMICs Early growth } \\
\text { faltering }\end{array}$ & $\begin{array}{l}\text { Loss in } \\
\text { education } \\
\text { attainment }\end{array}$ & $\begin{array}{l}\text { Loss in } \\
\text { economic } \\
\text { growth }\end{array}$ & $\begin{array}{l}\text { Human capital losses are largest in } \\
\text { South Asia due to stunting }\end{array}$ \\
\hline TGP & $\begin{array}{l}\text { The Global } \\
\text { Panel on } \\
\text { Agriculture } \\
\text { and Food } \\
\text { Systems for } \\
\text { Nutrition }\end{array}$ & 2016 & $\begin{array}{l}\text { Pakistan, India and } \\
\text { Bangladesh were } \\
\text { mentioned particularly } \\
\text { when the report writes } \\
\text { on Asian countries. } \\
\text { Investment in human } \\
\text { capital, actions for } \\
\text { better maternal and } \\
\text { child nutrition }\end{array}$ & $\begin{array}{l}\text { Forgone } \\
\text { economic } \\
\text { growth both of } \\
\text { individual and } \\
\text { country }\end{array}$ & $\begin{array}{l}\text { Decline in } \\
\text { global economy }\end{array}$ & $\begin{array}{l}\text { Stunting, vitamin and mineral } \\
\text { deficiencies together result in losses } \\
\text { of up to } 3 \% \text { of GDP in low-income } \\
\text { developing countries including South } \\
\text { Asian countries. }\end{array}$ \\
\hline Jessica Fanzo & $\begin{array}{l}\text { Journal of } \\
\text { International } \\
\text { Affairs }\end{array}$ & 2014 & $\begin{array}{l}\text { Asian Country including } \\
\text { India. } \\
\text { Long-term insufficient } \\
\text { nutrients intake and } \\
\text { frequent infections } \\
\text { before } 2 \text { years of age }\end{array}$ & $\begin{array}{l}\text { Delayed motor } \\
\text { development, } \\
\text { impaired } \\
\text { cognition and } \\
\text { poor school } \\
\text { performance }\end{array}$ & Poverty & $\begin{array}{l}\text { SDG's goal of reducing stunting } \\
\text { depends on the government strategy } \\
\text { and favorable environment. }\end{array}$ \\
\hline
\end{tabular}




\begin{tabular}{|c|c|c|c|c|c|c|}
\hline Author's Name & Publication & $\begin{array}{l}\text { Year of } \\
\text { publication }\end{array}$ & $\begin{array}{l}\text { Studied Country/ies } \\
\text { Prevailing } \\
\text { Circumstances }\end{array}$ & Outcomes & $\begin{array}{l}\text { Effect on } \\
\text { Economic } \\
\text { growth }\end{array}$ & Focus View \\
\hline $\begin{array}{l}\text { J. Hoddinott et } \\
\text { al. }\end{array}$ & $\begin{array}{l}\text { Maternal and } \\
\text { Child } \\
\text { Nutrition, } \\
\text { Wiley }\end{array}$ & 2013 & $\begin{array}{l}\text { Developing countries } \\
\text { and India, Pakistan, } \\
\text { Bangladesh, Bhutan, } \\
\text { Nepal. Stunting, low age } \\
\text { at first birth and more } \\
\text { pregnancies }\end{array}$ & $\begin{array}{l}\text { Low test } \\
\text { scores and } \\
\text { nonverbal } \\
\text { cognitive } \\
\text { disorders, } \\
\text { lower } \\
\text { household per } \\
\text { capita } \\
\text { expenditure } \\
\text { and higher } \\
\text { poverty }\end{array}$ & $\begin{array}{l}\text { Adverse } \\
\text { consequences } \\
\text { on human, } \\
\text { social and } \\
\text { economic } \\
\text { capital }\end{array}$ & $\begin{array}{l}\text { Summary of repercussions of stunting } \\
\text { in the initial } 1000 \text { days including } \\
\text { enneadic months in mother's womb till } \\
\text { the old age and cost-benefit ratios of } \\
\text { nutritional interventions to reduce } \\
\text { stunting. }\end{array}$ \\
\hline PRB & $\begin{array}{l}\text { Population } \\
\text { Reference } \\
\text { Bureau }\end{array}$ & 2012 & $\begin{array}{l}\text { India and Senegal. } \\
\text { 1-Intervention in proper } \\
\text { nutrition } \\
\text { 2-High income/ } \\
\text { economic growth }\end{array}$ & $\begin{array}{l}\text { 1-better } \\
\text { performance } \\
\text { in school, } \\
\text { higher lifetime } \\
\text { earnings and } \\
\text { increased } \\
\text { productivity in } \\
\text { labor force } \\
\text { 2-Wealthier } \\
\text { population, } \\
\text { higher } \\
\text { purchasing } \\
\text { power and } \\
\text { more essential } \\
\text { nutrients }\end{array}$ & $\begin{array}{l}\text { 1-effective } \\
\text { interventions } \\
\text { 2-better health } \\
\text { and larger } \\
\text { increase in } \\
\text { economic } \\
\text { growth }\end{array}$ & $\begin{array}{l}\text { There is more to stunting than just } \\
\text { economic growth and that is lack of } \\
\text { action in interventions and poor } \\
\text { strategy by the policymakers }\end{array}$ \\
\hline Milman et al. & $\begin{array}{l}\text { American } \\
\text { Society for } \\
\text { Nutritional } \\
\text { Sciences }\end{array}$ & 2005 & $\begin{array}{l}\text { Bangladesh India, } \\
\text { Nepal, Pakistan, Sri } \\
\text { Lanka, Bhutan } 85 \\
\text { countries. } \\
\text { Reduced stunting by } \\
\text { long-term and specified } \\
\text { interventions }\end{array}$ & $\begin{array}{l}\text { Reduced } \\
\text { stunting } \\
\text { prevalence }\end{array}$ & $\begin{array}{l}\text { Increase in } \\
\text { country's } \\
\text { economic } \\
\text { performance }\end{array}$ & $\begin{array}{l}\text { Child stunting is associated with } \\
\text { impact over long-term development } \\
\text { and specific interventions needed to } \\
\text { curb it }\end{array}$ \\
\hline $\begin{array}{l}\text { M. Shekar et } \\
\text { al. }\end{array}$ & $\begin{array}{l}\text { Maternal \& } \\
\text { Child } \\
\text { Nutrition, } \\
\text { Wiley }\end{array}$ & 2016 & $\begin{array}{l}\text { South Asia including } \\
\text { India. } \\
\text { 1-Stunting } \\
\text { 2-Intervention in } \\
\text { nutrition }\end{array}$ & $\begin{array}{l}\text { 1-increased } \\
\text { mortality, } \\
\text { morbidity, } \\
\text { decrease } \\
\text { cognitive } \\
\text { abilities, poor } \\
\text { education } \\
\text { outcomes and } \\
\text { loss in } \\
\text { earnings } \\
\text { 2-Reduced } \\
\text { poverty }\end{array}$ & $\begin{array}{l}\text { 1-Loss in } \\
\text { national } \\
\text { economic } \\
\text { productivity } \\
\text { 2-High } \\
\text { economic } \\
\text { growth }\end{array}$ & $\begin{array}{l}\text { Stunting can result in loss of earnings } \\
\text { leading to losses to national economic } \\
\text { productivity. }\end{array}$ \\
\hline $\begin{array}{l}\text { Rudra \& } \\
\text { Kurian }\end{array}$ & $\begin{array}{l}\text { Observer } \\
\text { Research } \\
\text { Foundation }\end{array}$ & 2017 & $\begin{array}{l}\text { India. } \\
\text { Stunting }\end{array}$ & $\begin{array}{l}\text { Hindrances in } \\
\text { physical and } \\
\text { mental } \\
\text { development } \\
\text { high risk of } \\
\text { diabetes, } \\
\text { obesity and } \\
\text { hypertension } \\
\text { in future }\end{array}$ & $\begin{array}{l}\text { Reduced } \\
\text { earnings }\end{array}$ & $\begin{array}{l}\text { Stunting has permanent and } \\
\text { irreversible consequences leading to } \\
\text { poor performance in school leaning } \\
\text { and lesser earnings in adulthood. }\end{array}$ \\
\hline $\begin{array}{l}\text { Pakistan } \\
\text { Scaling Up } \\
\text { Nutrition and } \\
\text { United Nations } \\
\text { World Food } \\
\text { Programme }\end{array}$ & $\begin{array}{l}\text { United } \\
\text { Nations } \\
\text { World Food } \\
\text { Programme }\end{array}$ & 2017 & $\begin{array}{l}\text { Pakistan. } \\
\text { Malnutrition }\end{array}$ & $\begin{array}{l}\text { Loss of } \\
\text { laborer, higher } \\
\text { healthcare } \\
\text { expenses and } \\
\text { lower } \\
\text { productivity }\end{array}$ & Low GDP & $\begin{array}{l}\text { The consequences of poor nutrition - } \\
\text { includes lost laborers, increase } \\
\text { healthcare expenses and lesser } \\
\text { productivity that costs Pakistan } \\
\text { US\$7.6 billion, or } 3 \text { percent of GDP } \\
\text { every year }\end{array}$ \\
\hline Shreya Raman & IndiaSpend & 2018 & $\begin{array}{l}\text { India. } \\
\text { Childhood stunting }\end{array}$ & $\begin{array}{l}\text { Impaired brain } \\
\text { development, } \\
\text { lower cognitive } \\
\text { and socio- } \\
\text { emotional skill, } \\
\text { lower } \\
\text { attainment of } \\
\text { schooling }\end{array}$ & $\begin{array}{l}\text { Lesser earnings } \\
\text { by workforce } \\
\text { and reduced } \\
\text { per-capita } \\
\text { income }\end{array}$ & $\begin{array}{l}\text { South Asia is topping the list in the } \\
\text { WHO Report on stunting that the } \\
\text { present working force is earning } 10 \% \\
\text { lesser because they are stunted in } \\
\text { childhood. }\end{array}$ \\
\hline
\end{tabular}




\begin{tabular}{|c|c|c|c|c|c|c|}
\hline Author's Name & Publication & $\begin{array}{l}\text { Year of } \\
\text { publication }\end{array}$ & $\begin{array}{l}\text { Studied Country/ies } \\
\text { Prevailing } \\
\text { Circumstances }\end{array}$ & Outcomes & $\begin{array}{l}\text { Effect on } \\
\text { Economic } \\
\text { growth }\end{array}$ & Focus View \\
\hline $\begin{array}{l}\text { McGoovern. et } \\
\text { al }\end{array}$ & $\begin{array}{l}\text { International } \\
\text { Journal of } \\
\text { Epidemiology }\end{array}$ & 2017 & $\begin{array}{l}\text { South Asian Countries } \\
\text { and Africa including } \\
\text { SAARC countries India. } \\
\text { Childhood stunting }\end{array}$ & Poverty & $\begin{array}{l}\text { Reduced } \\
\text { economic } \\
\text { growth }\end{array}$ & $\begin{array}{l}\text { Summarizes the pathway linking } \\
\text { stunting and economic growth. The } \\
\text { preferred studies state that } 1-\mathrm{cm} \\
\text { increase in stature with respect to } \\
\text { height is associated with a } 4 \% \text { higher } \\
\text { wages in men and a } 6 \% \text { higher wage } \\
\text { in women. }\end{array}$ \\
\hline $\begin{array}{l}\text { Subramanian } \\
\& \\
\text { Subramanyam }\end{array}$ & $\begin{array}{l}\text { Journal of } \\
\text { Korean } \\
\text { Medical } \\
\text { Science }\end{array}$ & 2015 & $\begin{array}{l}\text { India. } \\
\text { Undernourishment }\end{array}$ & Poverty & $\begin{array}{l}\text { Lack in } \\
\text { strategic } \\
\text { intervention }\end{array}$ & $\begin{array}{l}\text { Evidence from study on India along } \\
\text { with other developing countries shows } \\
\text { that economic growth has little to no } \\
\text { impact on increasing child's nutritional } \\
\text { level. }\end{array}$ \\
\hline $\begin{array}{l}\text { S.V. } \\
\text { Subramanian } \\
\text { et al }\end{array}$ & $\begin{array}{l}\text { Maternal \& } \\
\text { Child } \\
\text { Nutrition, } \\
\text { Wiley }\end{array}$ & 2016 & $\begin{array}{l}\text { India. } \\
\text { Child growth faltering }\end{array}$ & $\begin{array}{l}\text { Benefits in } \\
\text { cognitive and } \\
\text { economic } \\
\text { development }\end{array}$ & $\begin{array}{l}\text { Macroeconomic } \\
\text { growth }\end{array}$ & $\begin{array}{l}\text { Summary of different approaches to } \\
\text { reduce stunting from India. Integrated } \\
\text { and structural factors policy } \\
\text { suggestions. }\end{array}$ \\
\hline W. Joe et al. & $\begin{array}{l}\text { Maternal \& } \\
\text { Child } \\
\text { Nutrition, } \\
\text { Wiley }\end{array}$ & 2016 & $\begin{array}{l}\text { India. } \\
\text { 1-public development } \\
\text { expenditure } \\
\text { 2-increase in aggregate } \\
\text { income poverty level }\end{array}$ & $\begin{array}{l}\text { Failure in } \\
\text { reduced child } \\
\text { undernutrition }\end{array}$ & $\begin{array}{l}\text { Lack of } \\
\text { planning in } \\
\text { intervention }\end{array}$ & $\begin{array}{l}\text { Macroeconomic growth didn't } \\
\text { translate into maternal and child } \\
\text { health and reduction in poverty. }\end{array}$ \\
\hline
\end{tabular}

3.2 Evidence from intervention studies and natural experiment:

Table 3. shows two intervention studies. One study by (8) in 34 developing countries another by (38) from India showed better performance in height when given balanced protein-calorie supplementation to pregnant women and children under 6 years. The intervention group was $14 \mathrm{~mm}$ taller than the control group, with a $\mathrm{p}$-value of less than $1 \%$, i.e., $\mathrm{p}=0.007$. The distribution of nutrients of iodized salt, iron foliate, calcium, energy protein, vitamin $\mathrm{A}$, and zinc to pregnant women and toddlers for ten years, along with the promotion of breastfeeding, supplemental feeding, complementary food education, and treatment of severe acute malnutrition, gave a cost-benefit ratio of 1:5-1:6 in Hyderabad, India (8).

A natural experiment in Table 4. by (39) from 86 developing economies, including India, found that both agrarian (agriculture) and non-agrarian (industrial and services) growth reduce stunting prevalence. A $10 \%$ increase in agrarian GDP per capita reduces child stunting by $9.6 \%$, and a $10 \%$ increase in non-agrarian GDP per capita reduces non-agrarian growth by $8.4 \%$. 
Table 3

Evidence from intervention studies

\begin{tabular}{|c|c|c|c|c|c|c|c|c|c|c|}
\hline $\begin{array}{l}\text { Author's } \\
\text { Name }\end{array}$ & $\begin{array}{l}\text { Publication } \\
\& \text { Year of } \\
\text { publication }\end{array}$ & $\mathbf{N}$ & Location & $\begin{array}{l}\text { Study } \\
\text { design }\end{array}$ & Duration & Gender & $\begin{array}{l}\text { Nutritional } \\
\text { status }\end{array}$ & Impact & Outcomes & $\begin{array}{l}\text { Effect c } \\
\text { Econon } \\
\text { growth }\end{array}$ \\
\hline $\begin{array}{l}\text { Emanuela } \\
\text { Galasso } \\
\text { \& Adam } \\
\text { Wagstaff }\end{array}$ & $\begin{array}{l}2019 ; \\
\text { Economics } \\
\text { and } \\
\text { Human } \\
\text { Biology; } \\
\text { Elsevier }\end{array}$ & $\begin{array}{l}34 \\
\text { developing } \\
\text { countries }\end{array}$ & $\begin{array}{l}34 \\
\text { developing } \\
\text { countries }\end{array}$ & Intervention & 10 years & $\begin{array}{l}\text { Females, } \\
\text { and } \\
\text { children } \\
\text { from } \\
\text { both } \\
\text { gender }\end{array}$ & Poor & Stunting & $\begin{array}{l}\text { Lesser } \\
\text { schooling } \\
\text { and lower } \\
\text { cognition }\end{array}$ & $\begin{array}{l}\text { Lower F } \\
\text { capita } \\
\text { income }\end{array}$ \\
\hline
\end{tabular}

\begin{tabular}{|c|c|c|c|c|c|c|c|c|c|c|}
\hline S. Kinra & 2008; BMJ & 1165 & $\begin{array}{l}29 \text { villages } \\
\text { (15 } \\
\text { intervention, } \\
14 \text { control) } \\
\text { Near } \\
\text { Hyderabad, } \\
\text { South India }\end{array}$ & Intervention & $\begin{array}{l}15 \text { years' follow- } \\
\text { up of } \\
\text { participants } \\
\text { born within an } \\
\text { earlier } \\
\text { controlled, } \\
\text { community trial } \\
\text { of nutritional } \\
\text { supplementation } \\
\text { integrated with } \\
\text { other public } \\
\text { health programs }\end{array}$ & Both & Poo & $\begin{array}{l}\text { Integrated } \\
\text { nutritional } \\
\text { supplemen } \\
\text { along with } \\
\text { parallel } \\
\text { public } \\
\text { health } \\
\text { programs }\end{array}$ & $\begin{array}{l}\text { Taller } \\
\text { controlled } \\
\text { group }\end{array}$ & $\begin{array}{l}\text { Better } \\
\text { econor } \\
\text { perform }\end{array}$ \\
\hline
\end{tabular}

Table 4

Evidence from natural experiment

\begin{tabular}{|c|c|c|c|c|c|c|c|c|c|c|}
\hline $\begin{array}{l}\text { Author's } \\
\text { Name }\end{array}$ & $\begin{array}{l}\text { Publication } \\
\& \text { Year of } \\
\text { publication }\end{array}$ & $\mathbf{N}$ & Location & $\begin{array}{l}\text { Study } \\
\text { design }\end{array}$ & Duration & Impact & Outcomes & $\begin{array}{l}\text { Effect on } \\
\text { Economic } \\
\text { growth }\end{array}$ & Gender & Result \\
\hline $\begin{array}{l}\text { Mary et } \\
\text { al }\end{array}$ & $\begin{array}{l}\text { 2019; Wiley; } \\
\text { Development } \\
\text { Policy } \\
\text { Review }\end{array}$ & 367 & $\begin{array}{l}86 \\
\text { developing } \\
\text { countries }\end{array}$ & $\begin{array}{l}\text { Natural } \\
\text { IV }\end{array}$ & $\begin{array}{l}1984- \\
2014\end{array}$ & $\begin{array}{l}\text { Growth } \\
\text { in } \\
\text { agrarian } \\
\text { and } \\
\text { non- } \\
\text { agrarian } \\
\text { sectors }\end{array}$ & $\begin{array}{l}\text { Food } \\
\text { security }\end{array}$ & $\begin{array}{l}\text { Reduced } \\
\text { child } \\
\text { stunting }\end{array}$ & Both & $\begin{array}{l}\text { Both agrarian and non-agrarian } \\
\text { growth decrease stunting, } \\
\text { agrarian growth is significantly } \\
\text { superior to non-agrarian growth } \\
\text { in this regard. The estimated } \\
\text { impacts is decrease in } 9.6 \% \text { in } \\
\text { stunting due to } 10 \% \text { increase } \\
\text { GDP for agrarian (as opposed to } \\
8.4 \% \text { for non-agrarian) }\end{array}$ \\
\hline
\end{tabular}

3.3 Evidence from prospective studies:

Table 5. shows data from prospective studies. One prospective cohort study (a cohort study is a study where participants are enrolled before developing the outcome of the researcher's interest) was conducted on malnutrition and economic expansion in two economically resurgent states, Bihar and Gujarat. Gujarat improved its record in combating malnutrition, reducing undernutrition from 73.04 percent in 2007 to 25.09 percent in 2013 , as the state's economy grew. 
While Bihar's economy grew by $8 \%$ in 2012 , undernutrition remained at $82 \%$. This 10 -yearlong study reveals that substantial economic growth does not alleviate malnutrition. Four of the seven prospective studies were conducted in India only. Two were undertaken in various underdeveloped nations, with India included in the SAARC cohort. One (16) was conducted in Pakistan for 55 years, giving evidence that the sprinkles receiving group had a greater income than those in the placebo receiving group after 4 months of getting homemade food rich in zinc and iron. Other study reveals that harm in the first two years of a child's life can lead to persistent handicap and that this child can potentially affect future generation/s (11). Stunted children are more prone to accidents, have higher absenteeism from school and work (40), and have lower physical energy for farming and industrial labor $(41,42)$. (7) estimated that birth length is associated with adult height and postnatal growth is associated with education achieved, which effects income. 
Table 5

Evidence from prospective studies

\begin{tabular}{|c|c|c|c|c|c|c|c|c|c|c|}
\hline Author's Name & $\begin{array}{l}\text { Publication } \\
\& \text { Year of } \\
\text { publication }\end{array}$ & $\mathbf{N}$ & Location & Study design & Duration & Gender & $\begin{array}{l}\text { Nutritional } \\
\text { status }\end{array}$ & Impacts & $\begin{array}{l}\text { Economic } \\
\text { Outcomes }\end{array}$ & Reas \\
\hline Ruia et al. & $\begin{array}{l}2018, \\
\text { Indian } \\
\text { Journal of } \\
\text { Community } \\
\text { Medicine, } \\
\text { Wolters } \\
\text { Kluwer }\end{array}$ & $\begin{array}{l}2 \\
\text { States } \\
\text { from } \\
\text { India }\end{array}$ & $\begin{array}{l}\text { Bihar, India } \\
\& \text { Gujarat, } \\
\text { India }\end{array}$ & $\begin{array}{l}\text { Prospective } \\
\text { cohort. } \\
\text { An } \\
\text { exploratory } \\
\text { study }\end{array}$ & 10 years & $\begin{array}{l}\text { Females } \\
\text { and } \\
\text { children }\end{array}$ & Poor & $\begin{array}{l}\text { Integrated child } \\
\text { development } \\
\text { scheme }\end{array}$ & $\begin{array}{l}\text { No evidence } \\
\text { of excessive } \\
\text { growth }\end{array}$ & $\begin{array}{l}\text { Poor } \\
\text { impli }\end{array}$ \\
\hline $\begin{array}{l}\text { C. G. Victora, L. } \\
\text { Adair, C. Fall, P. } \\
\text { C. Hallal, R. } \\
\text { Martorell, L. } \\
\text { Richter, H. S. } \\
\text { Sachdev }\end{array}$ & $\begin{array}{l}\text { 2008, } \\
\text { Lancet }\end{array}$ & $\begin{array}{l}8181 \\
\text { for } \\
\text { India }\end{array}$ & $\begin{array}{l}\text { Brazil, } \\
\text { Guatemala, } \\
\text { India, the } \\
\text { Philippines, } \\
\text { and South } \\
\text { Africa }\end{array}$ & $\begin{array}{l}\text { prospective } \\
\text { cohort }\end{array}$ & $\begin{array}{l}\text { Recruited } \\
\text { between } \\
1969-72\end{array}$ & $\begin{array}{l}\text { Females } \\
\text { and } \\
\text { children }\end{array}$ & Average & $\begin{array}{l}\text { Mother and } \\
\text { child under- } \\
\text { nutrition }\end{array}$ & $\begin{array}{l}\text { School } \\
\text { delays, lower } \\
\text { income and } \\
\text { scarce } \\
\text { assets, low } \\
\text { birth weight, } \\
\text { lower body } \\
\text { mass, higher } \\
\text { glucose } \\
\text { concentration } \\
\text { and blood } \\
\text { pressure }\end{array}$ & Adve \\
\hline $\begin{array}{l}\text { Satyanarayana } \\
\text { et al. }\end{array}$ & 1977 & & India & Prospective & $1960-63$ & Males & Poor & Malnourishment & $\begin{array}{l}\text { Accidents } \\
\text { and } \\
\text { absenteeism }\end{array}$ & $\begin{array}{l}\text { Decre } \\
\text { econı } \\
\text { outpı }\end{array}$ \\
\hline
\end{tabular}

\begin{tabular}{|c|c|c|c|c|c|c|c|c|c|}
\hline $\begin{array}{l}\text { Satyanarayana } \\
\text { et al. }\end{array}$ & 1979 & 1480 & India & Prospective & $\begin{array}{l}\text { In1965 it } \\
\text { was an } \\
\text { ongoing } \\
\text { study }\end{array}$ & Males & Poor & Malnutrition & $\begin{array}{l}\text { Reduced } \\
\text { physical } \\
\text { capacity in } \\
\text { farming }\end{array}$ \\
\hline
\end{tabular}

\begin{tabular}{|c|c|c|c|c|c|c|c|c|c|c|}
\hline $\begin{array}{l}\text { K. } \\
\text { Satyanarayana } \\
\text { et al. }\end{array}$ & 1984 & 57 & India & Prospective & 1977 & $\begin{array}{l}\text { Males } \\
\text { between } \\
20-35\end{array}$ & Adequate & Malnutrition & $\begin{array}{l}\text { Reduced } \\
\text { physical } \\
\text { capacity in } \\
\text { industry }\end{array}$ & $\begin{array}{l}\text { Decre } \\
\text { econı } \\
\text { outpı }\end{array}$ \\
\hline Stein et al. & $\begin{array}{l}\text { 2013; The } \\
\text { Journal of } \\
\text { pediatrics }\end{array}$ & 4518 & $\begin{array}{l}\text { Brazil, } \\
\text { Guatemala, } \\
\text { India, the } \\
\text { Philippines, } \\
\text { and South } \\
\text { Africa }\end{array}$ & $\begin{array}{l}\text { Prospective } \\
\text { Cohort }\end{array}$ & $\begin{array}{l}15-18 \\
\text { years in } \\
\text { different } \\
\text { countries }\end{array}$ & Both & & $\begin{array}{l}\text { Preterm or small } \\
\text { for gestation } \\
\text { age birth }\end{array}$ & $\begin{array}{l}\text { Persistent } \\
\text { deficit in } \\
\text { height and } \\
\text { schooling }\end{array}$ & $\begin{array}{l}\text { Decre } \\
\text { econı } \\
\text { activi } \\
\text { adult }\end{array}$ \\
\hline
\end{tabular}




\begin{tabular}{|c|c|c|c|c|c|c|c|c|c|c|}
\hline Author's Name & $\begin{array}{l}\text { Publication } \\
\& \text { Year of } \\
\text { publication }\end{array}$ & $\mathbf{N}$ & Location & Study design & Duration & Gender & $\begin{array}{l}\text { Nutritional } \\
\text { status }\end{array}$ & Impacts & $\begin{array}{l}\text { Economic } \\
\text { Outcomes }\end{array}$ & Reas \\
\hline $\begin{array}{l}\text { W. Sharieff, S. } \\
\text { H., Zlotkin, M. } \\
\text { D. Krahn et al. }\end{array}$ & 2008 & 5000 & $\begin{array}{l}\text { Karachi, } \\
\text { Pakistan }\end{array}$ & $\begin{array}{l}\text { Retrospective } \\
\text { cohort to see } \\
\text { a cost-benefit } \\
\text { analysis }\end{array}$ & $\begin{array}{l}4 \\
\text { months } \\
\text { and } \\
\text { followed } \\
\text { for } 55 \\
\text { years in } \\
\text { a Markov } \\
\text { process } \\
\text { of time } \\
\text { cycles. }\end{array}$ & Both & Poor & $\begin{array}{l}\text { Intervention } \\
\text { through zinc } \\
\text { and iron in } \\
\text { home-made } \\
\text { foods }\end{array}$ & $\begin{array}{l}\text { Reduction in } \\
\text { diarrhea, } \\
\text { improved } \\
\text { hemoglobin } \\
\text { level }\end{array}$ & $\begin{array}{l}\text { Redu } \\
\text { child } \\
\text { mort } \\
\text { high } \\
\text { and } \\
\text { lifetir } \\
\text { earni }\end{array}$ \\
\hline
\end{tabular}

Source: Author's construction

3.4 Evidence from adult height and age by quasi-experiment and linear regression:

As tabulated in Table 6. one quasi-experiment was found, that involved five SAARC countries: Afghanistan, Bangladesh, India, Nepal, and Pakistan. The study used a backward-looking accounting framework to estimate how much a country's per capita income is lower today because some of its workforce was stunted as a child, and a forward-looking accounting framework to estimate the net present values of the costs and benefits of a package of interventions aimed at reducing childhood stunting (8). The study projected that implementing the study by $(9,43)$ could save developing countries between $5 \%$ and $7 \%$ per capita GDP.

Table 7. shows the wage-height relationship. According to the findings of $(44,45)$ on household survey data in 12 developing countries (including Pakistan and Nepal), consistent income development could lead to a significant decrease in stunting. Research by $(17,18,22)$ found no link between macroeconomic growth and child stunting, nor did macroeconomic growth lead to a reduction in poverty. According to (46), a 10\% rise in GDP reduces child stunting prevalence by $2.7 \%$, while a percentage point increase in child stunting results in a $0.4 \%$ loss in GDP per capita. According to (46), stunting reduces annual per capita GDP by 13.5 percent. A study by (47) identified a link between $\$ 1 /$ day poverty and a 0.62 prevalence of stunting.

Table 6

Evidence from adult height and age by quasi-experiment

\begin{tabular}{|c|c|c|c|c|c|c|c|c|c|c|}
\hline $\begin{array}{l}\text { Author's } \\
\text { Name }\end{array}$ & $\begin{array}{l}\text { Publication } \\
\& \text { Year of } \\
\text { publication }\end{array}$ & $N$ & Location & Data & Study design & Age & Impact & Outcomes & $\begin{array}{l}\text { Economic } \\
\text { effects }\end{array}$ & Gender \\
\hline $\begin{array}{l}\text { Emanuela } \\
\text { Galasso } \\
\text { \& Adam } \\
\text { Wagstaff }\end{array}$ & $\begin{array}{l}2019 ; \\
\text { Economics } \\
\text { and } \\
\text { Human } \\
\text { Biology; } \\
\text { Elsevier }\end{array}$ & $\begin{array}{l}34 \\
\text { developing } \\
\text { countries, } \\
\text { including } \\
\text { SAARC } \\
\text { Countries }\end{array}$ & $\begin{array}{l}34 \\
\text { developing } \\
\text { countries }\end{array}$ & $\begin{array}{l}\text { World } \\
\text { Development } \\
\text { Indicators } \\
\text { (WDI) }\end{array}$ & $\begin{array}{l}\text { Development } \\
\text { accounting } \\
\text { approach }\end{array}$ & $\begin{array}{l}\text { Backward- } \\
\text { looking } \\
\text { exercise } \\
\text { of the } \\
\text { workforce } \\
\text { who are } \\
50 \text { at the } \\
\text { time of } \\
\text { study }\end{array}$ & $\begin{array}{l}\text { Nutrition } \\
\text { intervention }\end{array}$ & $\begin{array}{l}\text { Increase in } \\
\text { schooling } \\
\text { years and } \\
\text { cognitive } \\
\text { development }\end{array}$ & $\begin{array}{l}\text { Increase } \\
\text { in GDP }\end{array}$ & Both \\
\hline
\end{tabular}

Source: Author's construction 
Table 7

Evidence from adult height and age by linear regression

\begin{tabular}{|c|c|c|c|c|c|c|c|c|}
\hline Author's Name & $\begin{array}{l}\text { Publication \& } \\
\text { Year of } \\
\text { publication }\end{array}$ & $N$ & Location & Data & Study design & Age & $\begin{array}{l}\text { Economic } \\
\text { Impact }\end{array}$ & Outcomes \\
\hline Haddad et al & $\begin{array}{l}\text { 2002, } \\
\text { Economic } \\
\text { Development } \\
\text { and Cultural } \\
\text { Change }\end{array}$ & 179 & $\begin{array}{l}63 \\
\text { developing } \\
\text { countries }\end{array}$ & $\begin{array}{l}\text { World Health } \\
\text { Organization, } \\
\text { ACC/SCN, } \\
\text { World Bank, } \\
\text { Food and } \\
\text { Agriculture } \\
\text { Organization } \\
\text { (FAO), UNESCO }\end{array}$ & OLS & $\begin{array}{l}1970-96 . \\
\text { Woman and } \\
\text { children under } \\
5 \text { years of age }\end{array}$ & $\begin{array}{l}\text { Increase in per- } \\
\text { capita income }\end{array}$ & $\begin{array}{l}\text { Improved } \\
\text { child nutritic } \\
\text { level }\end{array}$ \\
\hline $\begin{array}{l}\text { Smith \& } \\
\text { Haddad }\end{array}$ & $\begin{array}{l}\text { 2003, Oxford } \\
\text { University } \\
\text { Press }\end{array}$ & $\begin{array}{l}12 \text { developing } \\
\text { countries, } 61 \\
\text { developing } \\
\text { economies for } \\
\text { cross country } \\
\text { estimations }\end{array}$ & $\begin{array}{l}12 \\
\text { countries } \\
\text { including } \\
\text { Pakistan } \\
\text { and Nepal }\end{array}$ & $\begin{array}{l}\text { WHO, ACC/SCN, } \\
\text { World Bank }\end{array}$ & OLS \&IV & $\begin{array}{l}\text { Household } \\
\text { survey }\end{array}$ & $\begin{array}{l}\text { Increase in } \\
\text { household } \\
\text { income due to } \\
\text { increase in } \\
\text { national income }\end{array}$ & $\begin{array}{l}\text { Reduction in } \\
\text { malnutrition }\end{array}$ \\
\hline $\begin{array}{l}\text { Subramanyam } \\
\text { et al. }\end{array}$ & $\begin{array}{l}\text { 2011, } \\
\text { Economic } \\
\text { Growth and } \\
\text { Child } \\
\text { Undernutrition }\end{array}$ & $\begin{array}{l}28,066 \text {, } \\
26,121 \text {, and } \\
23,139 \\
\text { respectively } \\
\text { with } \\
\text { corresponding } \\
\text { dates. }\end{array}$ & $\begin{array}{l}\text { Cross- } \\
\text { section } \\
\text { waves } \\
1992-93, \\
1998-99 \\
\text { and 2005- } \\
\text { 06, India }\end{array}$ & $\begin{array}{l}\text { NFHS was } \\
\text { conducted in } \\
\text { India. The } \\
\text { NFHS is part of } \\
\text { the } \\
\text { Demographic } \\
\text { and Health } \\
\text { Surveys (DHS), } \\
\text { Reserve Bank of } \\
\text { India }\end{array}$ & OLS & $\begin{array}{l}\text { Children } \\
\text { undue } 5 \text { years } \\
\text { of age }\end{array}$ & $\begin{array}{l}\text { Increase in per- } \\
\text { capita income }\end{array}$ & $\begin{array}{l}\text { Reduction in } \\
\text { child under- } \\
\text { nutrition }\end{array}$ \\
\hline $\begin{array}{l}\text { Rasmus } \\
\text { Heltberg }\end{array}$ & $\begin{array}{l}\text { 2009; Health } \\
\text { Economics, } \\
\text { Wiley }\end{array}$ & $\begin{array}{l}\text { Developing } \\
\text { countries }\end{array}$ & $\begin{array}{l}\text { Developing } \\
\text { countries }\end{array}$ & $\begin{array}{l}\text { World } \\
\text { Development } \\
\text { Indicators. }\end{array}$ & OLS & & $\begin{array}{l}\text { Economic } \\
\text { growth }\end{array}$ & $\begin{array}{l}\text { Very small } \\
\text { decrease in } \\
\text { child } \\
\text { malnutrition }\end{array}$ \\
\hline $\begin{array}{l}\text { Sebastien } \\
\text { Mary }\end{array}$ & $\begin{array}{l}\text { 2018, MDPI, } \\
\text { Economies }\end{array}$ & $\begin{array}{l}74 \text { developing } \\
\text { countries } \\
\text { observed } \\
\text { between } 1984 \\
\text { and } 2014\end{array}$ & $\begin{array}{l}74 \\
\text { developing } \\
\text { countries }\end{array}$ & $\begin{array}{l}\text { Political Risk } \\
\text { Services (PRS), } \\
\text { World Health } \\
\text { Organization, } \\
\text { World Bank } \\
\text { database, } \\
\text { Climate } \\
\text { Research Unit } \\
\text { (CRU) and the } \\
\text { Tyndall Centre } \\
\text { for Climate } \\
\text { Change } \\
\text { Research (TYN) }\end{array}$ & OLS & $\begin{array}{l}\text { Children } \\
\text { undue } 5 \text { years } \\
\text { of age born } \\
\text { between 1984- } \\
2014\end{array}$ & $\begin{array}{l}\text { Economic } \\
\text { growth }\end{array}$ & $\begin{array}{l}\text { Reduced chi } \\
\text { stunting }\end{array}$ \\
\hline
\end{tabular}




\begin{tabular}{|c|c|c|c|c|c|c|c|c|}
\hline Author's Name & $\begin{array}{l}\text { Publication \& } \\
\text { Year of } \\
\text { publication }\end{array}$ & $N$ & Location & Data & Study design & Age & $\begin{array}{l}\text { Economic } \\
\text { Impact }\end{array}$ & Outcomes \\
\hline Vollmer et al. & $\begin{array}{l}\text { 2014; Lancet } \\
\text { Global Health }\end{array}$ & 462,854 & $\begin{array}{l}36 \\
\text { developing } \\
\text { countries }\end{array}$ & $\begin{array}{l}\text { Demographic } \\
\text { and Health } \\
\text { Surveys. }\end{array}$ & OLS & 0-35 months, & $\begin{array}{l}\text { Macroeconomics } \\
\text { growth }\end{array}$ & $\begin{array}{l}\text { Early } \\
\text { childhood } \\
\text { malnutrition }\end{array}$ \\
\hline & & & & $\begin{array}{l}\text { Penn World } \\
\text { Tables } 8.0\end{array}$ & & & & \\
\hline $\begin{array}{l}\text { William Joe, } \\
\text { Ramaprasad } \\
\text { Rajaram and } \\
\text { S. V. } \\
\text { Subramanian }\end{array}$ & $\begin{array}{l}\text { 2016, Wiley, } \\
\text { Maternal \& } \\
\text { Child } \\
\text { Nutrition }\end{array}$ & & India & $\begin{array}{l}\text { National Family } \\
\text { Health Surveys } \\
\text { (NFHS) of India, } \\
\text { UNICEF, Central } \\
\text { Statistical } \\
\text { Organization of } \\
\text { the Ministry of } \\
\text { Statistics and } \\
\text { Program } \\
\text { Implementation, } \\
\text { Government of } \\
\text { India, Reserve } \\
\text { Bank of India }\end{array}$ & $\begin{array}{l}\text { OLS \& } \\
\text { Alternative } \\
\text { model } \\
\text { specifications }\end{array}$ & $\begin{array}{l}\text { State-wise } \\
\text { prevalence of } \\
\text { developmental } \\
\text { indicators, } \\
\text { Indian States } \\
1992-1993 \\
\text { and } 2005- \\
2006\end{array}$ & $\begin{array}{l}\text { 1-public } \\
\text { development } \\
\text { expenditure } \\
\text { 2-increase in } \\
\text { aggregate } \\
\text { income poverty } \\
\text { level }\end{array}$ & $\begin{array}{l}\text { Failure in } \\
\text { reduced chil } \\
\text { undernutritic }\end{array}$ \\
\hline
\end{tabular}

Source: Author's construction

\section{4- Discussion}

4.1 Summary of the evidence linking childhood stunting to economic outcomes in SAARC region

Several studies have discovered an inverse relationship between SAARC economic growth and child stunting. Nutritional deficiencies (maternal and child), stunting genes, lack of education (including literacy and maternal health during pregnancy and nursing), wrong eating habits, poor hygiene conditions, frequent infections in children, the role of paternal stimulus, poverty, macroeconomic shocks, and lack of government expenditure or moderate pro-poor policies are variables that contributed to the region's child stunting (17-20, 22, 29, 36, 37). Stunting negatively affects a child's academic, physical, mental, and motor abilities, lowering income, employment, and wages $(11,12,19,28-30,35)$

Studies comparing height and wages demonstrate a link. Studies conducted in other countries (no studies were found on SAARC), validate our claim that taller people earn more. Four studies partly on height and wage India, were found. In one study, providing pregnant women with a balanced protein calorie supplement raised their baby's height by $14 \mathrm{~mm}$ (38). A $1-\mathrm{cm}$ increase in stature relative to height leads to a $4 \%$ rise in male and a $6 \%$ increase in female earnings (5). In both agriculture and industry, shorter workers produced poor output (40-42). An established relationship between birth length, adult height, and schooling (7) was found.

Hunger, undernutrition, and poverty during pregnancy or in the first 1000 days affect adult income. A nutritious diet has a cost-benefit ratio of 1.5-1.6 in lowincome countries (8). A study of 8181 people indicated that hunger can affect a child's life, from schooling to adulthood income (11). A lack of nutrition hampers individual output in the industrial and agricultural sectors (40-42). Malnutrition costs Pakistan \$7.6 billion per year (3\% of GDP) (30). India's workforce earns $10 \%$ less due to the fact that they were stunted in childhood (34).

\subsection{Interpreting the findings of Stunting to Economic Growth}

A country's human capital is priceless and is measured by health and education. Human capital that is healthier and more efficient grows the economy faster. Similarly, unhealthier and less skilled human capital is more likely to become a burden. This inefficient productivity issue impacts a country's economy. Stunting, if it occurs in childhood, affects a person's health and productivity in adulthood. Expenses for diminished immunity, chronic illness, health drift to the next generation, and other variables might further harm a person's financial situation. 
However, while the SAARC region's human capital ranks among the top 10 out of 195 countries, there remains a wide range of differences in human productivity level (21). Stunting, vitamin and mineral deficiencies cost low-income developing countries, particularly in South Asia, up to $3 \%$ of their GDP (30). Stunting causes the highest human capital losses in this region and can have irrevocable, lasting implications that can limit economic growth, resulting in the decrease of the country's GDP. Stunting reduces per capita income by 5-7\% (8). Childhood stunting has been linked to reduced school attendance. This might be attributed to poor motor and cognitive skills, frequent infections and illnesses, chronic illness, and other stunting-related difficulties. Adults with less education and health difficulties have a harder time finding work in a competitive economy. If they do get jobs, they are more likely to be injured and have inferior physical work capacity $(11,40-42)$. OLS estimation shows that a $1 \%$ increase in stunting reduces GDP by $0.4 \%$, which has a $13.5 \%$ impact on developing countries' GDP (46).

\subsection{Interpreting the findings of Economic Growth to Stunting}

As evidenced by industrialized countries, faster economic growth reduces stunting. Economists attribute this to increasing GDP per capita. Macroeconomic shocks affect incomes, which affect generations, and so on. In natural trials, a 10\% rise in agricultural GDP reduces stunting prevalence by $9.6 \%$, while a similar increase in non-agricultural GDP reduces it by $8.4 \%$ (39). Increasing national GDP improves child nutrition, and a $10 \%$ rise in GDP reduces stunting by $2.7 \%(39,46,47)$. While many studies demonstrate that an increase in GDP reduces stunting, few shows that it has no or just a minor impact. The authors claim that a rise in GDP has little or no influence due to a gap in the government or policymakers' transition mechanisms $(17,18,20)$. Institutions trying to reduce stunting prevalence must enhance strategies and interventions, so that growth in GDP or national income should not have any or limited influence. Moreover, policies must target a larger number of poor people.

4.4 Strengths and weaknesses of this review

Our systematic review's methodical approach is its strength. We couldn't find any other reviews like it, so it's a unique addition to the literature. The lack of research on SAARC and policymakers' lack of understanding of the difficulties occurring in this region, which are distinct from other geographic areas where stunting is common. This assessment has focused on the SAARC region and can help policymakers plan policies and initiatives to minimize stunting prevalence. This review used a comprehensive search approach that covered five worldwide databases, including all study and intervention categories. We utilized methodological appraisal scores to assess each study's quality. The review followed PRISMA guidelines. Our review was assessed using the Mixed Method Assessment Tool (MMAT) to reduce bias.

The review has limitations. Our exploratory evaluation did not exclude papers based on low quality scores. To find more relevant content, we could have searched other worldwide academic databases and search engines, but we were time-constrained. It's also possible that some discoveries have become obsolete. Studies in other languages are excluded, causing language bias. Therefore, we utilized Google Scholar and Chrome to reduce bias in our search. There has been little research undertaken on emerging countries' having one or more SAARC members along with other developing countries. This review used such studies to draw conclusions. So it was difficult to draw general conclusions based just on SAARC outcomes.

4.5 Implications for countries with high stunting prevalence

Children's health is often improved by increased per capita national affluence. Policymakers must examine how state policies affect policy effectiveness and child nutrition. Despite the fact that stunting is common in some areas, there is little understanding of how it affects adulthood and future earnings. Growing wealth disparities have impeded economic growth, causing problems for current and future generations. To decrease stunting, we need to have improved knowledge of prenatal and postnatal growth constraints. Improvements in child nutrition and health require equitable economic growth, not growth equality. Investing in pro-poor programmes and making food affordable. Primary care programmes are critical in times of economic crisis or food shortages and should be addressed quickly. Due to a lack of spillover effects, the transition channel through which macroeconomic growth is expected to positively influence poverty has proven unsuccessful. Inclusionary macroeconomic growth with active transition channels is required to reduce stunting.

\section{5- Conclusion}

The majority of quantitative evidence supports our hypothesis that stunting and economic outcomes are inversely correlated. Economic growth should have a spillover effect. Children who are healthy and have no history of stunting do better in school and earn more than their stunted peers. Not much can be attained in outcomes through nutritional, fortification, and micronutrient interventions unless tailored activities are done, therefore, better transition mechanism is needed. Direct health interventions have dramatically reduced stunting. Improving maternal health and interventions is also important in lowering stunting.

From conception to two years of age, children must be fed a balanced, healthy diet. For such children, better health will allow them to keep their average origin height (according to the WHO, average height varies by location and origin; for example, Chinese people are shorter than Pakistanis and Indians). The review reveals that schooling and skills attainment are better in children who are not stunted at an early age. Stunted children are more chronically ill, prone to infections, injuries, and accidents. Their absenteeism is higher, resulting in low productivity.

Stunting is usually a rural phenomenon, and agricultural prosperity raises rural living conditions. Macroeconomic shocks impact child stunting as it impact food nutrition, health and education. A stunted population have a pro-cyclical effect in the economic drift.

\section{Abbreviations}

GDP Gross Domestic Product

LMICs Lower Middle-Income Countries 
MMAT Mixed Method Assessment Tool

OLS Ordinary Least Square

PICO Patient/Population, Intervention, Comparison and Outcomes

PRISMA Preferred Reporting Items for Systematic Reviews and Meta-Analyses

SAARC South Asian Association for Regional Cooperation

SDG Sustainable Development Goals

UNFP United Nations Population Fund

UNICEF The United Nations Children's Fund

WB The World Bank

WHO World Health Organization

\section{Declarations}

\section{Funding}

This research received no specific funding from any agency in the government, private, commercial, or not-for-profit sectors

Conflict of interests

It has been declared by the authors that they do not have any conflict interest.

Ethics approval and consent for publication

Not applied

Code availability: not applicable

Consent to participate: not applicable

Consent for publication: not applicable

Availability of Data and materials

All available data is within the paper

Authors' contributions

Conceptualization: MSN. AA.

Formal analysis: MSN. HA

Funding acquisition: not applicable

Investigation: MSN. AA. HA

Methodology: MSN. AA

Supervision: AA

Writing - original draft: MSN.

Writing - review \& editing: MSN. AA. HA. DN

\section{References}

1. WHO. The UNICEF/WHO/WB Joint Child Malnutrition Estimates (JME) group released new data for 2021. World Health Organization; 2021.

2. WHO. Global Nutrition Targets 2025 (2014). Stunting policy brief international.; 2014.

3. Fenske N, Burns J, Hothorn T, Rehfuess EA. Understanding child stunting in India: a comprehensive analysis of socio-economic, nutritional and environmental determinants using additive quantile regression. PloS one. 2013;8(11):e78692. 
4. Aguayo VM, Menon P. Stop stunting: improving child feeding, women's nutrition and household sanitation in South Asia. Wiley Online Library; 2016. p. 311.

5. McGovern ME, Krishna A, Aguayo VM, Subramanian S. A review of the evidence linking child stunting to economic outcomes. International journal of epidemiology. 2017;46(4):1171-91.

6. McDonald CM, Olofin I, Flaxman S, Fawzi WW, Spiegelman D, Caulfield LE, et al. The effect of multiple anthropometric deficits on child mortality: metaanalysis of individual data in 10 prospective studies from developing countries. The American journal of clinical nutrition. 2013;97(4):896-901.

7. Stein AD, Barros FC, Bhargava SK, Hao W, Horta BL, Lee N, et al. Birth Status, Child Growth, and Adult Outcomes in Low- and Middle-Income Countries. The Journal of Pediatrics. 2013;163(6):1740-6.e4.

8. Galasso E, Wagstaff A. The aggregate income losses from childhood stunting and the returns to a nutrition intervention aimed at reducing stunting. Economics \& Human Biology. 2019;34:225-38.

9. Black RE, Victora CG, Walker SP, Bhutta ZA, Christian P, De Onis M, et al. Maternal and child undernutrition and overweight in low-income and middleincome countries. The lancet. 2013;382(9890):427-51.

10. UNICEF. Stop Stunting in South Asia A Common Narrative on Maternal and Child Nutrition.; 2013.

11. Victora CG, Adair L, Fall C, Hallal PC, Martorell R, Richter L, et al. Maternal and child undernutrition: consequences for adult health and human capital. The lancet. 2008;371(9609):340-57.

12. Dewey KG, Begum K. Long-term consequences of stunting in early life. Maternal \& Child Nutrition. 2011;7(s3):5-18.

13. Martorell R, Khan LK, Schroeder DG. Reversibility of stunting: epidemiological findings in children from developing countries. European journal of clinical nutrition. 1994;48:S45-57.

14. UNICEF. Stop stunting in South Asia - Part 1-The costs of stunting to children and nations in the region 2018. [Available from: https://www.unicef.org/rosa/stories/stop-stunting-south-asia-part-1.

15. WB. World Bank. 2018. Available from: https://blogs.worldbank.org/endpovertyinsouthasia/it-s-time-end-malnutrition-south-asia.

16. Ruia A, Gupta RK, Bandyopadhyay G, Gupta RR. An analysis of integrated child development scheme performance in contributing to alleviation of malnutrition in two economically resurgent states. Indian journal of community medicine: official publication of Indian Association of Preventive \& Social Medicine. 2018;43(1):44.

17. Subramanyam MA, Kawachi I, Berkman LF, Subramanian S. Is economic growth associated with reduction in child undernutrition in India? PLoS medicine. 2011;8(3):e1000424.

18. Vollmer S, Harttgen K, Subramanyam MA, Finlay J, Klasen S, Subramanian SV. Association between economic growth and early childhood undernutrition: evidence from 121 Demographic and Health Surveys from 36 low-income and middle-income countries. The lancet global health. 2014;2(4):e225-e34.

19. Subramanian S, Subramanyam MA. Limits to economic growth: why direct investments are needed to address child undernutrition in India. Journal of Korean medical science. 2015;30(Suppl 2):S131-S3.

20. Joe W, Rajaram R, Subramanian S. Understanding the null-to-small association between increased macroeconomic growth and reducing child undernutrition in India: role of development expenditures and poverty alleviation. Maternal \& child nutrition. 2016;12:196-209.

21. Lim SS, Updike RL, Kaldjian AS, Barber RM, Cowling K, York H, et al. Measuring human capital: a systematic analysis of 195 countries and territories, 1990-2016. The Lancet. 2018;392(10154):1217-34.

22. Subramanian S, Mejía-Guevara I, Krishna A. Rethinking policy perspectives on childhood stunting: time to formulate a structural and multifactorial strategy. Maternal \& child nutrition. 2016;12:219-36.

23. Hong QN, Fàbregues S, Bartlett G, Boardman F, Cargo M, Dagenais P, et al. The Mixed Methods Appraisal Tool (MMAT) version 2018 for information professionals and researchers. Education for Information. 2018;34(4):285-91.

24. Dahan-Oliel N, Shikako-Thomas K, Majnemer A. Quality of life and leisure participation in children with neurodevelopmental disabilities: a thematic analysis of the literature. Quality of Life Research. 2012;21(3):427-39.

25. Akhlaq A, McKinstry B, Muhammad KB, Sheikh A. Barriers and facilitators to health information exchange in low-and middle-income country settings: a systematic review. Health policy and planning. 2016;31(9):1310-25.

26. Hoddinott J, Behrman JR, Maluccio JA, Melgar P, Quisumbing AR, Ramirez-Zea M, et al. Adult consequences of growth failure in early childhood. The American journal of clinical nutrition. 2013;98(5):1170-8.

27. Fink G, Peet E, Danaei G, Andrews K, McCoy DC, Sudfeld CR, et al. Schooling and wage income losses due to early-childhood growth faltering in developing countries: national, regional, and global estimates. The American Journal of Clinical Nutrition. 2016;104(1):104-12.

28. Rudra S, Kurian OC. Tracking SDG targets on health and nutrition: Challenges and opportunities for streamlining surveys: Observer Research Foundation; 2017.

29. Milman A, Frongillo EA, de Onis M, Hwang J-Y. Differential improvement among countries in child stunting is associated with long-term development and specific interventions. The Journal of Nutrition. 2005;135(6):1415-22.

30. UNFP. Malnutrition Costs Pakistan US\$7.6 Billion Annually, New Study Reveals. Pakistan Scaling Up Nutrition and United Nations World Food Programme 2017.

31. Batha E. Childhood stunting can cut future earnings by up to a quarter. Reuter; 2016.

32. (TGP) TGP. The cost of malnutrition. Why policy action is urgent. London, UK: Global Panel on Agriculture and Food Systems for Nutrition; 2016.

33. Ferreira FH, Schady N. Aggregate economic shocks, child schooling and child health. World Bank Policy Research Working Paper. 2008(4701). 
34. Raman S. 66\% Working Indians Earn Less Because Of Childhood Stunting, One Of World's Worst Rates. IndiaSpend; 2018.

35. Shekar M, Dayton Eberwein J, Kakietek J. The costs of stunting in South Asia and the benefits of public investments in nutrition. Maternal \& Child Nutrition. 2016;12:186-95.

36. Fanzo J. STUNTING: A COUNTRY'S LASTING BURDEN. Journal of International Affairs. 2014;67(2):165-73.

37. PRB. Combating Malnutrition With More Than Income Growth. 2012.

38. Kinra S, Sarma KR, Mendu VVR, Ravikumar R, Mohan V, Wilkinson IB, et al. Effect of integration of supplemental nutrition with public health programmes in pregnancy and early childhood on cardiovascular risk in rural Indian adolescents: long term follow-up of Hyderabad nutrition trial. Bmj. $2008 ; 337$.

39. Mary S, Shaw K, Paloma SGY. Does the sectoral composition of growth affect child stunting reductions? Development Policy Review. 2019;37(2):225-44.

40. Satyanarayana K, Naidu AN, Chatterjee B, Rao BN. Body size and work output. The American journal of clinical nutrition. 1977;30(3):322-5.

41. Satyanarayana K, Naidu AN, Narasinga Rao B. Nutritional deprivation in childhood and the body size, activity, and physical work capacity of young boys. The American journal of clinical nutrition. 1979;32(9):1769-75.

42. Satyanarayana K, Naidu AN, Rau BN. Nutrition, physical work capacity and work output. Human Growth and Development: Springer; 1984 . p. 535-40.

43. Ahmed T, Hossain M, Mahfuz M, Choudhury N, Hossain MM, Bhandari N, et al. Severe Acute Malnutrition in Asia. Food and Nutrition Bulletin. 2014;35(2_suppl1):S14-S26.

44. Haddad L, Alderman H, Appleton S, Song L, Yohannes Y. Reducing child malnutrition: How far does income growth take us? The World Bank Economic Review. 2003;17(1):107-31.

45. Smith LC, Haddad L. How potent is economic growth in reducing undernutrition? What are the pathways of impact? New cross-country evidence. Economic Development and Cultural Change. 2002;51(1):55-76.

46. Mary S. How much does economic growth contribute to child stunting reductions? Economies. 2018;6(4):55.

47. Heltberg R. Malnutrition, poverty, and economic growth. Health Economics. 2009;18(S1):S77-S88.

\section{Figures}

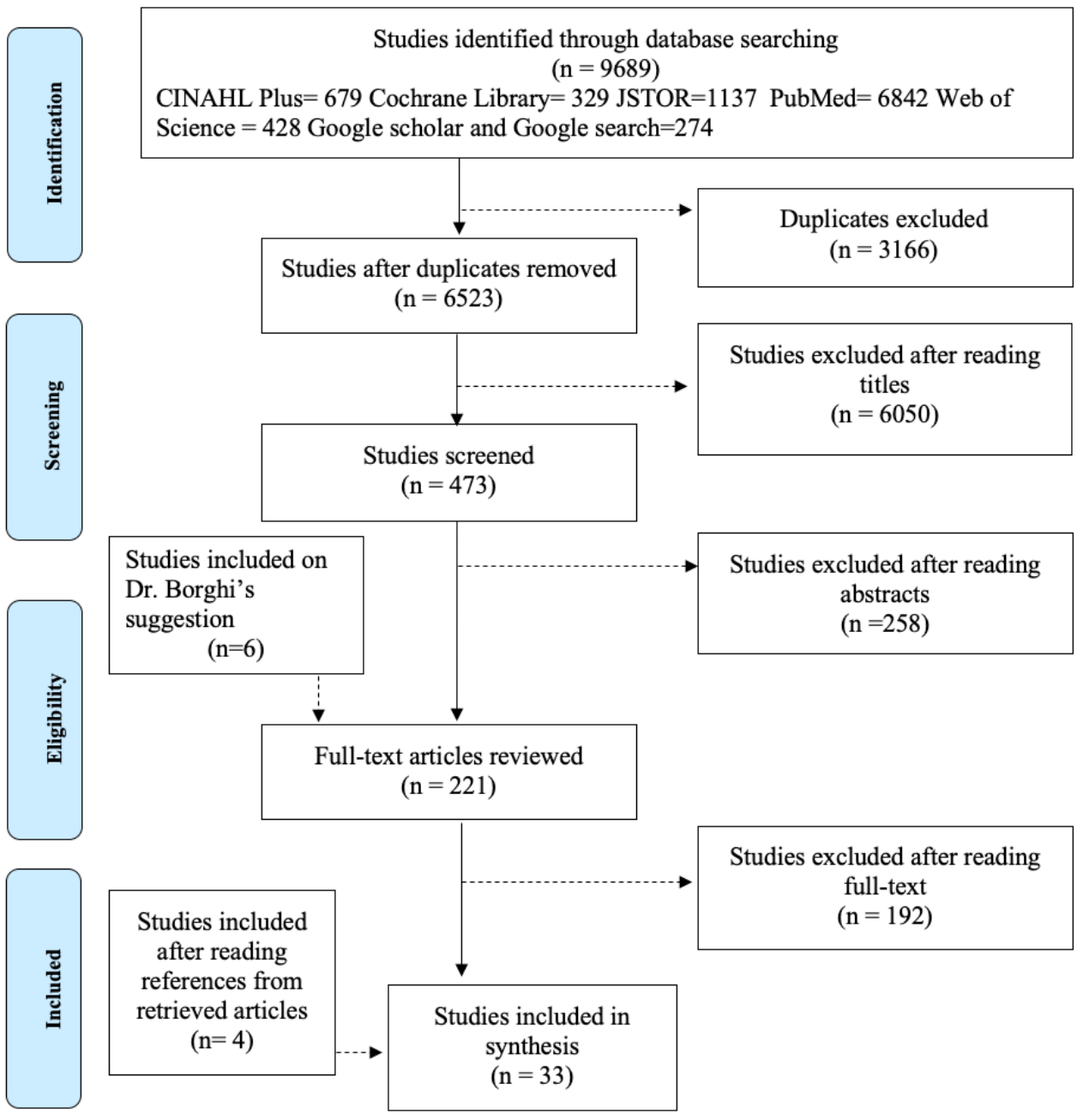

Source: Author's construction using PRISMA standard flow chart

Figure 1 
PRISMA flow chart

\section{Supplementary Files}

This is a list of supplementary files associated with this preprint. Click to download.

- Appendix.docx 